\title{
Development of Spontaneous Recurrent Seizures after Kainate-Induced Status Epilepticus
}

\author{
Philip A. Williams, ${ }^{1}$ Andrew M. White, ${ }^{2,3}$ Suzanne Clark, ${ }^{1}$ Damien J. Ferraro, ${ }^{1}$ Waldemar Swiercz, ${ }^{2,3}$ Kevin J. Staley, ${ }^{2,3}$ \\ and F. Edward Dudek ${ }^{1}$ \\ ${ }^{1}$ Department of Biomedical Sciences, Colorado State University, Fort Collins, Colorado 80523, and Departments of ${ }^{2}$ Pediatrics and ${ }^{3}$ Neurology, University of \\ Colorado Health Sciences Center, Denver, Colorado 80262
}

Acquired epilepsy (i.e., after an insult to the brain) is often considered to be a progressive disorder, and the nature of this hypothetical progression remains controversial. Antiepileptic drug treatment necessarily confounds analyses of progressive changes in human patients with acquired epilepsy. Here, we describe experiments testing the hypothesis that development of acquired epilepsy begins as a continuous process of increased seizure frequency (i.e., proportional to probability of a spontaneous seizure) that ultimately plateaus. Using nearly continuous surface cortical and bilateral hippocampal recordings with radiotelemetry and semiautomated seizure detection, the frequency of electrographically recorded seizures (both convulsive and nonconvulsive) was analyzed quantitatively for $\sim 100 \mathrm{~d}$ after kainate-induced status epilepticus in adult rats. The frequency of spontaneous recurrent seizures was not a step function of time (as implied by the "latent period"); rather, seizure frequency increased as a sigmoid function of time. The distribution of interseizure intervals was nonrandom, suggesting that seizure clusters (i.e., short interseizure intervals) obscured the early stages of progression, and may have contributed to the increase in seizure frequency. These data suggest that (1) the latent period is the first of many long interseizure intervals and a poor measure of the time frame of epileptogenesis, (2) epileptogenesis is a continuous process that extends much beyond the first spontaneous recurrent seizure, (3) uneven seizure clustering contributes to the variability in occurrence of epileptic seizures, and (4) the window for antiepileptogenic therapies aimed at suppressing acquired epilepsy probably extends well past the first clinical seizure.

Key words: convulsion; EEG; epilepsy; latent period; radiotelemetry; seizure clusters

\section{Introduction}

Epilepsy is generally divided into genetic and acquired forms; acquired epilepsy is thought to arise from a brain insult (e.g., status epilepticus) and generally occurs after a latent period. Temporal lobe epilepsy (TLE) is an important form of acquired epilepsy that usually includes mesial temporal sclerosis (Margerison and Corsellis, 1966) with complex partial seizures that may spread secondarily to cause convulsive seizures. The time between a brain insult and onset of clinical seizures (i.e., the latent period) is highly variable, ranging from months to years (French et al., 1993; Mathern et al., 1995; Annegers et al., 1998). Antiepileptic drug therapy is typically initiated when a patient first reports seizures. The reliance on self-reporting of seizures from patients with postseizure amnesia, the lack of continuous electro-

\footnotetext{
Received Oct. 2, 2008; revised Dec. 29, 2008; accepted Jan. 5, 2009.

This work was supported by National Institutes of Health Grant NS045144.

Correspondence should be addressed to Dr. F. Edward Dudek, Department of Physiology, University of Utah School of Medicine, 420 Chipeta Way, Suite 1700, Salt Lake City, UT 84108-1297. E-mail: ed.dudek@hsc.utah.edu.

P. A. Williams's present address: Department of Neuroscience, Case Western Reserve University, 10900 Euclid Avenue, Cleveland, $0 \mathrm{H} 44106$.

S. Clark's present address: School of Pharmacy, University of Wyoming, Laramie, WY 82071.

D. J. Ferraro's present address: Department of Molecular Endocrinology, Merck, Inc., West Point, PA 19486.

W. Swiercz and K. J. Staley's present address: Department of Neurology, Massachusetts General Hospital, Harvard Medical School, 55 Fruit Street, Boston, MA 02114.

D01:10.1523/JNEUROSCI.0980-08.2009

Copyright $\odot 2009$ Society for Neuroscience $\quad$ 0270-6474/09/292103-10\$15.00/0
}

encephalographic (EEG) monitoring, and the use of therapy to suppress seizures have prevented quantitative studies on the natural history of epileptogenesis in humans. Animal models with spontaneous recurrent seizures may provide insights into the temporal features of acquired epilepsy.

The latent period is generally considered to be the time when a brain insult induces molecular and cellular mechanisms that lead to spontaneous recurrent seizures, and is thus considered a measure of epileptogenesis (Walker et al., 2002). The presence of a latent period, and the apparent variability or unpredictability of the subsequent spontaneous recurrent seizures, has led to the view that epileptogenesis occurs only during the time between the insult and the first clinical seizure. Therefore, development of epilepsy is sometimes represented as a "step function" of time after brain injury (Fig. 1A). Many other studies have suggested that the development of acquired epilepsy can be progressive, at least initially (Bertram and Cornett, 1993, 1994; French et al., 1993; Mathern et al., 1995; Hellier et al., 1998; Tasch et al., 1999; Fuerst et al., 2001; Gorter et al., 2001; Wuarin and Dudek, 2001). Epileptogenesis clearly occurs during the latent period, but another hypothesis states that the end of the latent period (i.e., the first clinical seizure) is not a terminal milestone for the mechanisms of epileptogenesis; rather, the process of epileptogenesis is a continuous function of time that persists long after the first clinical seizure. A corollary to this hypothesis is that a prolonged 
$1 \mathrm{~A}$

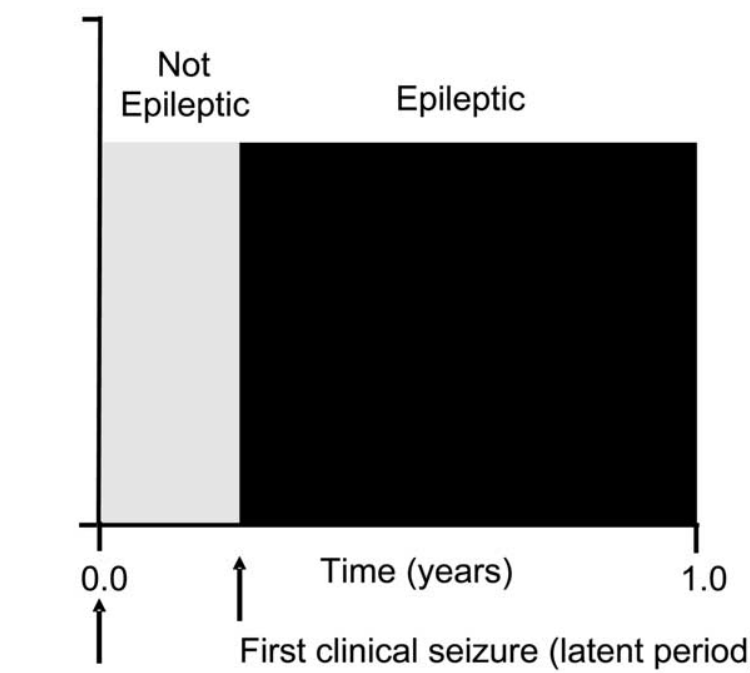

Brain insult
1B

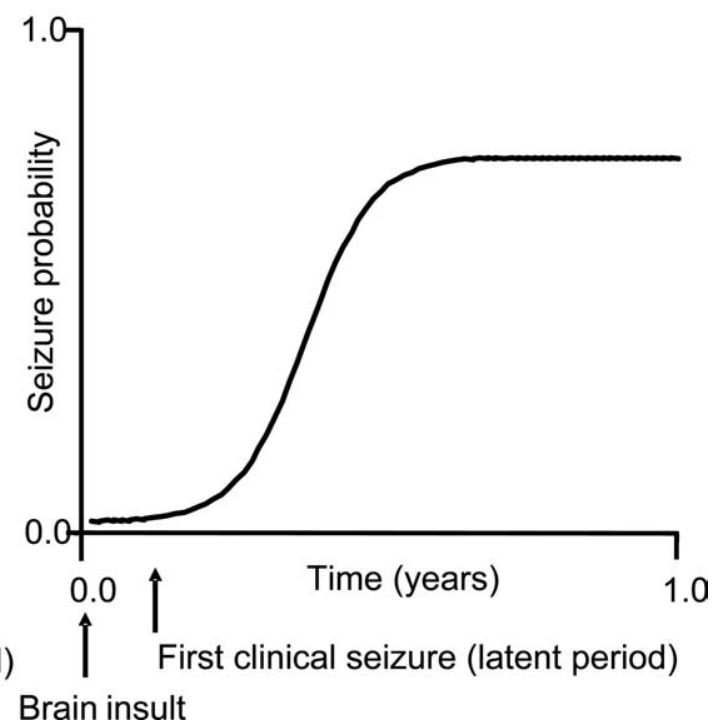

Figure 1. Graphical description of the step function $(\boldsymbol{A})$ and continuous-function $(\boldsymbol{B})$ hypotheses of the time course of epileptogenesis. The step function hypothesis describes two states: the first state is a seizure-free period that occurs after the initial brain insult (i.e., the latent period; not epileptic), and the second state is a period with seizures (i.e., epileptic). An important functional implication of the step function hypothesis is that the underlying causes of or mechanisms responsible for the generation of seizures are mature when the first seizure is observed, and the seizure rate immediately achieves a steady state or is variable. The continuous-function hypothesis states that seizure probability increases continuously after a brain insult; when considered as a sigmoid function of time after injury, the hypothesis predicts a gradual increase in seizure frequency or probability, followed by a period with an exponential increase, and then the gradual development of a steady state. In the continuous-function hypothesis, those factors that influence the generation of seizures and that control seizure rate are not fully developed at the time of the appearance of the first or "sentinel" seizure, and thus the seizure rate continuously increases with time as those processes mature.

period of increasing seizure probability is hypothetically manifest as a gradual increase in seizure frequency during acquired epileptogenesis and that a sigmoid function of seizure frequency versus time after the brain insult best represents acquired epileptogenesis (Fig. $1 B$ ). This alternative hypothesis would define the latent period as a probabilistic concept and predicts that the latent period is only the first of several long interseizure intervals.

The data in this study (using the repeated, low-dose kainate model of acquired epilepsy) suggest a new perspective on the latent period and provide evidence that acquired epileptogenesis is a continuous or progressive function of time in which clusters of seizures may obscure the continuous nature of the increase in seizure frequency, which may be quite difficult to detect without prolonged and continuous electrographic monitoring.

\section{Materials and Methods}

Terminology. All of the seizures in these experiments were recorded electrographically with radiotelemetry and behaviorally with video monitoring. "Electrographic (EEG) seizures" in this study refer to any seizure that was recorded electrically, which was virtually all of the seizures. These electrographically recorded seizures could either be "nonconvulsive seizures" or "convulsive motor seizures." A modified Racine scale (Racine, 1972; Ben-Ari, 1985) was used to characterize convulsive motor seizure severity as follows: class III seizures were defined by forelimb clonus and a lordotic posture; class IV seizures included forelimb clonus and rearing; class V seizures displayed a class IV seizure with loss of the righting reflex. All convulsive motor seizures were associated with electrographic (EEG) seizure activity, and would be considered to be generally similar to a "clinical seizure" in a human patient. The behaviors during nonconvulsive seizures were essentially those typically described as Racine (1972) class I or class II seizures; because characteristic electrographic seizures (see Fig. 2) were used to identify these nonconvulsive seizures, they are often described here as "electrographic nonconvulsive seizures" and could be a model for complex partial seizures. The "EEG-seizure latent period" was the time from the day of kainate treatment to the first elec- trographically recorded seizure, which was typically a nonconvulsive seizure. The "motor-seizure latent period" was the time to the first convulsive motor seizure, which was equal to (one case) or longer than the EEG-seizure latent period.

Surgery. Male Sprague Dawley rats (Harlan) weighing 180-200 g were kept under $12 \mathrm{~h}$ light/dark conditions throughout this study. All procedures in this study were approved by the Institutional Animal Care and Use Committee of Colorado State University. A detailed description of the surgery and recording techniques can be found in the study by Williams et al. (2006). Intrahippocampal electrodes were placed bilaterally in the granule cell layer. The dentate gyrus was chosen as a target for chronic intracranial electrographic recording of seizures because of the pathological and electrographic evidence suggesting that the dentate gyrus is involved in the seizures of patients with TLE, and because an extensive in vivo and in vitro database exists for this structure in rodent models of TLE, such as the kainate model (Margerison and Corsellis, 1966; Williamson et al., 1995) (for review, see Dudek et al., 2002, 2005; Dudek and Sutula, 2007; Sutula and Dudek, 2007). Briefly, the intrahippocampal electrodes were constructed from Teflon-coated steel wire (A-M Systems) and were implanted using stereotaxic coordinates (bilateral dorsal hippocampus; rostral-caudal, $-4.0 \mathrm{~mm}$; medial-lateral, $\pm 2.5 \mathrm{~mm}$; dorsal-ventral, $-3.3 \mathrm{~mm}$ at a bite of $-4.0 \mathrm{~mm}$ ); the position in the cell body layer was confirmed by increased spike activity, detected with an audio monitor. The dural recording electrode was placed on the left hemisphere $\sim 2 \mathrm{~mm}$ lateral and $1 \mathrm{~mm}$ rostral to the left intrahippocampal recording electrode [for details, see Williams et al. (2006)]. The dural reference electrodes were placed $\sim 1.5 \mathrm{~mm}$ caudal to the intrahippocampal recording electrodes.

The radiotelemetry unit (i.e., the three-channel PhysioTel Multiplus series transmitter F50-EEE; Transoma Medical) was positioned in a pocket created subcutaneously in the flank region through a $2 \mathrm{~cm}$ skin incision behind the scapula, and the leads tunneled forward to the rostral head incision. All electrodes were fixed in place with dental acrylic. The left and right intrahippocampal leads were connected to the male ends of the radiotelemetry unit, and then covered and fixed to the skull with dental acrylic. The local anesthetic bupivacaine $(0.4 \mathrm{ml} ; 7.5 \mathrm{mg} / \mathrm{ml})$ was 
applied liberally around the open cranial incision. Both incision sites were closed using 4-0 Dermalon (American Cyanamid). The animal was given $4-6 \mathrm{ml}$ of warmed Ringer's solution (subcutaneously), $0.06 \mathrm{ml}$ of buprenorphine $(0.1 \mathrm{mg} / \mathrm{kg}$, s.c.), removed from anesthesia, and placed back in its cage under a heat lamp for $30 \mathrm{~min}$. All animals were given 0.06 $\mathrm{ml}$ of buprenorphine $(0.01 \mathrm{mg} / \mathrm{kg}$, s.c.) and $0.2 \mathrm{ml}$ of penicillin $(300,000$ $\mathrm{IU}$, s.c.) for $3 \mathrm{~d}$ after surgery. The toenails were cut while the rats were anesthetized and, if necessary, once per week to decrease scratching and irritation at the incision sites. Rats were housed in individual cages within an isolated room after surgery and throughout the study and were given food and water ad libitum. For more details of this surgery, see Williams et al. (2006).

Electrographic recording with radiotelemetry. This study used the Dataquest A.R.T. Analog software from Transoma Medical, which is an analog acquisition system. The cages were placed on individual radio receiving plates (RPC-1; Transoma Medical), which captured data signals from the radiotransmitter and sent them to an input exchange matrix. The signal was then sent to the computer running Dataquest A.R.T. Analog software, which converted the digital output of the receiver into a calibrated analog output. Custom-made software (K. Staley, Boston, MA) was used to acquire the data with routines written in Visual Basic 6.0 (Microsoft). The electrographic data were written to DVD for analysis off-line.

Kainate treatment and subsequent animal care. Details of the kainate treatment, the response to kainate injections, and an analysis of status epilepticus have been previously published (Hellier et al., 1999; Dudek et al., 2005; Hellier and Dudek, 2005; Williams et al., 2006). One to 2 weeks after the implantation surgery, rats $(n=9)$ were injected with kainate $(5$ $\mathrm{mg} \cdot \mathrm{kg}^{-1} \cdot \mathrm{h}^{-1}$, i.p.; Sigma-Aldrich) diluted in sterile $0.9 \%$ saline at 2.5 $\mathrm{mg} / \mathrm{ml}$. Rats were continuously monitored for electrographic and convulsive motor seizures. Hourly kainate treatment continued in animals with convulsive seizures until class III, IV, or V seizures were evoked for at least $3 \mathrm{~h}$ (i.e., $>10$ convulsive seizures per hour). If an animal was nearing its endpoint, half-doses $(2.5 \mathrm{mg} / \mathrm{kg}$ ) were given to avoid excessive toxicity and mortality (for a review of this model, see Dudek et al., 2005). The mean total dose of kainate was $2.98 \pm 1.34 \mathrm{mg}$. Control animals $(n=$ 6 ) were treated with an equivalent volume and number of injections of sterile saline. All rats were given 3-6 $\mathrm{ml}$ of lactated Ringer's (subcutaneously) and apple slices after treatment. Subcutaneous fluids were continued for 2-3 dif the animals appeared lethargic. Kainate-induced seizures were complete by $24 \mathrm{~h}$, and the animals were eating and drinking within 24-36 $\mathrm{h}$ after kainate treatment.

Status epilepticus. During kainate treatment, the number and duration of each convulsive seizure was measured. Convulsive seizure duration was measured from the start to the end of clonus and lordotic posturing; animals then typically resumed normal motor activity and righted themselves. The mean number of convulsive motor seizures during kainate treatment was $99.2 \pm 20.2$, and the mean amount of time in convulsive seizures was $66.3 \pm 25.2 \mathrm{~min}$. Neither the total number of convulsive motor seizures during treatment nor the total amount of time in status epilepticus was correlated to the dose of kainate $\left(r^{2}=-0.124, p=0.56\right.$; $r^{2}=-0.125, p=0.56$, respectively).

EEG analysis. The seizure detection analysis was performed both visually (A. M. White) and in a semiautomated manner with custom-written software that minimized the potential for bias (for greater detail of the EEG analysis, see White et al., 2006). All of the electrographic recordings were examined for artifacts, and all of the electrographic seizures were confirmed by visual inspection. The investigators were not blinded to treatment or time after kainate-induced status epilepticus. Electroencephalographic seizures were differentiated from background noise by the appearance of large-amplitude (at least three times baseline), highfrequency (minimum of $5 \mathrm{~Hz}$ ) activity, with progression of the spike frequency (see Fig. 2) that lasted for a minimum of $30 \mathrm{~s}$. The behavioral data were used to confirm EEG seizure activity versus potential animalgenerated noise.

Video monitoring and analysis. Continuous videotaping of individually housed rats was accomplished using two Color Quad Observation Systems SOD14C4LN (Samsung). Time stamps for these systems were synchronized to the digitizing computer. Night recordings were performed with a Kodak 1A filter (Eastman Kodak) over a safelight, and daytime recordings with a diffuse fluorescent light. The behavioral data were used in this study for the determination of the latent period for convulsive motor seizures (vs nonconvulsive seizures) and for differentiating EEG seizure activity from electrical noise generated by jaw movement artifact or grooming.

Histological localization of recording sites. Similar previous studies with tethered recordings and perforant path stimulation using this same implantation method consistently revealed that the electrodes were located in the dentate gyrus of the hippocampus (Hellier et al., 1999). A histological examination of the hippocampi with cresyl violet staining for confirmation of hippocampal electrode placement showed that, in 8 of 15 rats (53\%), a linear or elliptical glial scar (a common indicator of recording site) was present in or near the dentate gyrus of septal hippocampal sections (supplemental Fig. 1, available at www.jneurosci.org as supplemental material). Aside from the glial scar, no evidence of damage from the recording electrodes was detected in any of the animals, although the expected histopathological alterations from the kainateinduced status epilepticus (Buckmaster and Dudek, 1997) were readily apparent.

Two related issues relating to the histological localization of the recording electrodes deserve consideration in terms of the reported conclusions from these studies. The first issue is the possibility that the intrahippocampal recording electrodes were not actually in the cell body layer of the dentate gyrus. Although it was not always possible to localize histologically the site of the intrahippocampal recording electrodes, all three electrodes (including the dural electrode) recorded a similar profile of electrographic (i.e., EEG) seizures throughout the recording period for all animals (see Fig. 2), thus indicating that the exact electrode placement was not an important factor in the data reported here. The second issue is whether the intrahippocampal electrodes induced sufficient damage to cause, or at least influence, the development of spontaneous recurrent seizures after kainate-induced status epilepticus. If damage from the recording electrodes (i.e., associated with the glial scars) caused nonconvulsive or convulsive electrographic seizures, then the control animals should have had seizures, because they also had glial scars; none of the control animals showed any seizures. Another issue was the site of seizure onset, which was not analyzed in this study. This question has been addressed in another model of status epilepticus-induced epilepsy, in which more recording electrodes were used (Bertram, 1997; Bertram et al., 1998). The exact time (and thus, site) of seizure onset can be difficult to determine (Bower and Buckmaster, 2008).

Statistical analysis. All graphs and statistical analyses were done using Prism software (GraphPad), except where noted. Means and distributions for each of the parameters and groups of parameters were determined. Differences among group means were tested for significance using ANOVA and post hoc tests (Student-Newman-Keuls multiplecomparisons test). Correlations were calculated using Pearson's $r$. Differences with $p \leq 0.05$ were considered statistically significant. A Poisson cumulative distribution function was generated with Microsoft Excel using the mean number of days for the slow growth, exponential, and plateau phases, and the mean interseizure intervals as the expected probability of seizure occurrence during that phase. These distributions were then compared with the cumulative probability functions for the actual interseizure intervals derived for the three different phases of seizure progression. A similar analysis was done with the generation of two ranges of random numbers that were matched to the total number of interseizure intervals for each animal during each phase of seizure progression, and limited by the total number of minutes for each individual animal's three different phases. Intervals were generated by taking the difference between the two ranges of randomly generated intervals. $\mathrm{Cu}-$ mulative distribution functions were then generated for the random intervals and compared with the cumulative distribution functions for the actual interseizure intervals. Tests for differences between cumulative distribution functions were performed with a Kolmogorov-Smirnov test, which was run using a program located at http://home.ubalt.edu/ ntsbarsh/Business-stat/otherapplets/ks.htm. 


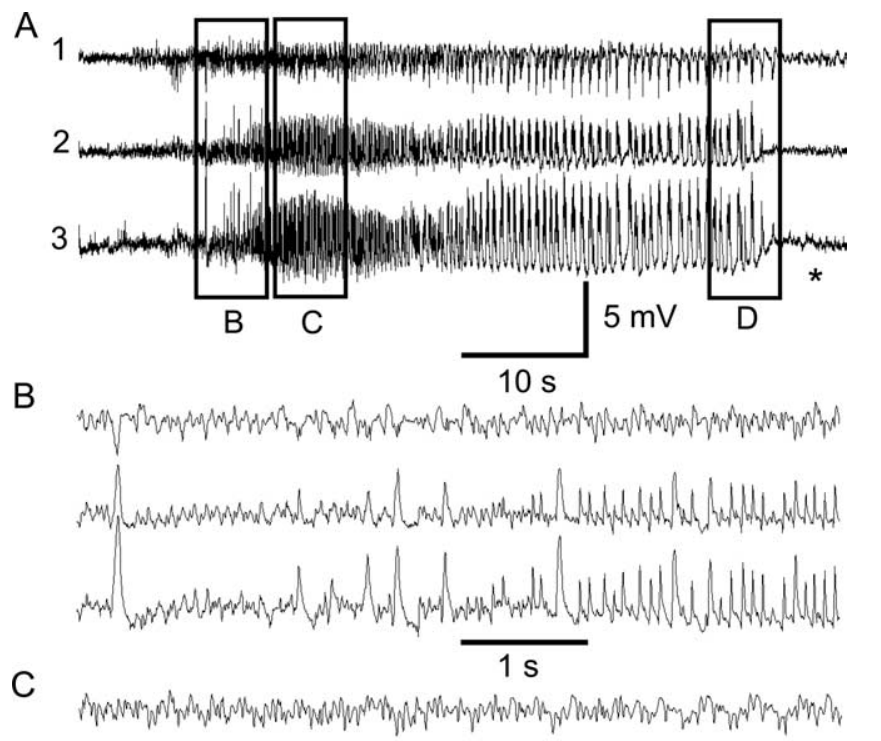

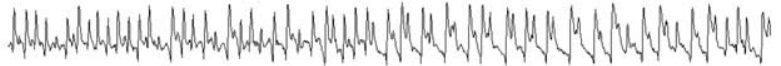

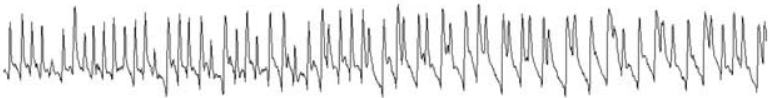

D

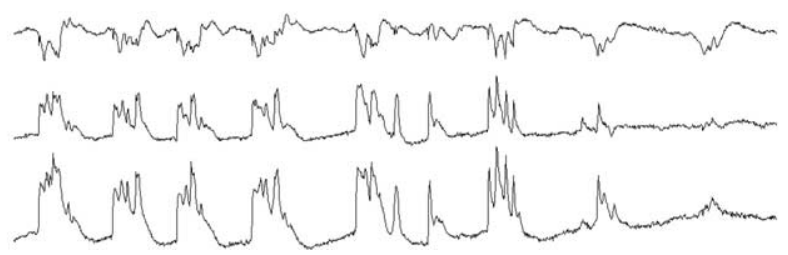

Figure 2. An electrographic seizure from a kainate-treated rat recorded with radiotelemetry. $\boldsymbol{A}$, These traces show an entire nonconvulsive seizure, which lasted $\sim 45 \mathrm{~s}$. Trace 1 corresponds to the surface recording at the level of the dura, trace 2 was from the left hippocampus, and trace 3 from the right hippocampus. $\boldsymbol{B}$, Seizure initiation with a single large EEG "spike" on all leads (i.e., based on the duration, this event and other "spikes" were presumably synchronous excitatory postsynaptic potentials, and not synchronous action potentials or "population spikes"), followed by high-amplitude high-frequency (i.e., $\geq 5 \mathrm{~Hz}$ ) EEG spike activity that signaled the beginning of the seizure. Note that the horizontal scale bar corresponds to a faster timescale. $\boldsymbol{C}$, Progression of the pattern from individual events into regular, high-frequency, large-amplitude EEG spikes (i.e., the tonic phase of the seizure). D, Activity near the termination of the seizure, with large-amplitude waves containing multiple EEG spikes (i.e., clonic phase), followed by a relative silent period ( $\boldsymbol{A}$, asterisk).

\section{Results}

\section{Latent period for electrographically recorded seizures}

Seven kainate-treated rats and six saline-treated rats were recorded for at least $100 \mathrm{~d}$, and two other kainate-treated rats were recoded for $<100 \mathrm{~d}$ (kainate-treated rats, $n=9$; saline controls, $n=6)$. Figure 2 shows an example of an electrographic recording of a spontaneous seizure. The mean and SD for the number of days to the first electrographically recorded seizure in the kainate-treated rats was $11.0 \pm 9.8 \mathrm{~d}$ with a median of $8 \mathrm{~d}$ and a range of $7-37 \mathrm{~d}$ (Figs. 3A, 4A). The high degree of variance in the latent period to the first electrographic seizure was attributable to a measurement of latent period from a single animal. The mean and SD for the electrographic-seizure latent period without this one value (i.e., a possible outlier) was $8.25 \pm 0.88 \mathrm{~d}$ with a median of $8 \mathrm{~d}$ and a range of 7-9 d. Aside from some of the earliest seizures, both hippocampal leads and the dural EEG lead (Fig. 2) showed qualitatively similar activation throughout the recording period for all kainate-treated rats. In all but one kainate-treated rat, the first electrographically recorded seizure was nonconvulsive. The saline-treated controls were recorded and studied the same as the kainate-treated rats, and no seizures (neither nonconvulsive nor convulsive seizures studied with both continuous video and EEG recordings) (see below) were detected in any of the animals in the control group. In five of the nine kainatetreated animals, one $24 \mathrm{~h}$ period (i.e., day 7) was not recorded between the first and the eighth day after kainate treatment. Thus, the true latent period (i.e., the time to the first seizure if all the time preceding the first seizure was known to be seizure free) for electrographically recorded seizures was in error by $<1 \mathrm{~d}$ for five of nine kainate-treated rats. No significant correlations between the total kainate dose during treatment or the total number of convulsive motor seizures observed during kainate treatment and the latent period for electrographic seizures were observed $\left(r^{2}=0.007, p=0.9\right.$, and $r^{2}=0.08, p=0.6$, respectively). These data indicate that the latent period for electrographic seizures was $\sim 1$ week (Figs. $3 A, 4 A$ ) for this model of TLE. Furthermore, the latent period to the first electrographic seizure was not detectably dependent on the severity of the status epilepticus.

\section{Latent period for convulsive motor seizures}

The mean number of days to the first observed convulsive motor or "clinical" seizure [i.e., a behavioral score of class III or above (Racine, 1972)] in all nine rats was $18.3 \pm 10.2 \mathrm{~d}$ with a median of $14.5 \mathrm{~d}$ and a range of $10-37 \mathrm{~d}$ (Fig. $4 A$ ). In only one of nine rats was the first electrographically recorded seizure a convulsive motor seizure, with a mean of $4.0 \pm 1.1$ electrographic nonconvulsive seizures occurring before the first convulsive motor seizure. A behavioral score was determined for the first observed convulsive motor seizure in all nine rats and the mode was a class $\mathrm{V}$ (i.e., class $\mathrm{V}$ is the most severe seizure type). When the video record of the first 20 electrographically recorded seizures was examined for each animal, $63 \%$ of the first 20 electrographic seizures across the nine animals were classified as convulsive motor seizures, and the mode of the behavioral scores for these convulsive motor seizures was also class $\mathrm{V}$. These data show that nonconvulsive electrographic seizures usually preceded convulsive motor seizures by $\sim 1$ week and that multiple nonconvulsive electrographic seizures almost always occurred before the appearance of the first convulsive motor seizure. This finding is similar to what has been shown previously by Bertram and Cornett (1993). Furthermore, in the kainate model, convulsive motor seizures tended to be severe once motor seizures occurred, and the convulsive motor seizures generally remained severe for the first 20 seizures and beyond.

\section{Progressive increase in seizure frequency}

To address the hypotheses that electrographically recorded seizures occur as a step function (Fig. $1 \mathrm{~A}$ ) or as a continuous function of time (Fig. $1 B$ ), the latent periods (for both the first electrographically recorded nonconvulsive seizure and the first convulsive motor seizure) were compared with the longest interseizure interval for the first 20 seizures (Fig. 4A). The successive interseizure intervals for the first 20 seizures were surprisingly variable and thus required a semilog plot to encompass the range of values (Fig. $4 B$ ). The mean and SD of the longest interseizure interval across all detected EEG seizures per animal was $4.36 \pm$ $2.65 \mathrm{~d}$, with a median of $4.1 \mathrm{~d}$ and a range of $0.94-8.5 \mathrm{~d}$ (Fig. $4 \mathrm{~A}$ ) (in this analysis, data from the periods without recordings were excluded). Because the longest interseizure intervals were $\sim 4-8$ $\mathrm{d}$ (Fig. $4 \mathrm{~B}$ ), these interseizure intervals were only slightly less than the latent periods to the first electrographically recorded nonconvulsive seizures (Fig. 4A). Although a positive trend was present, 


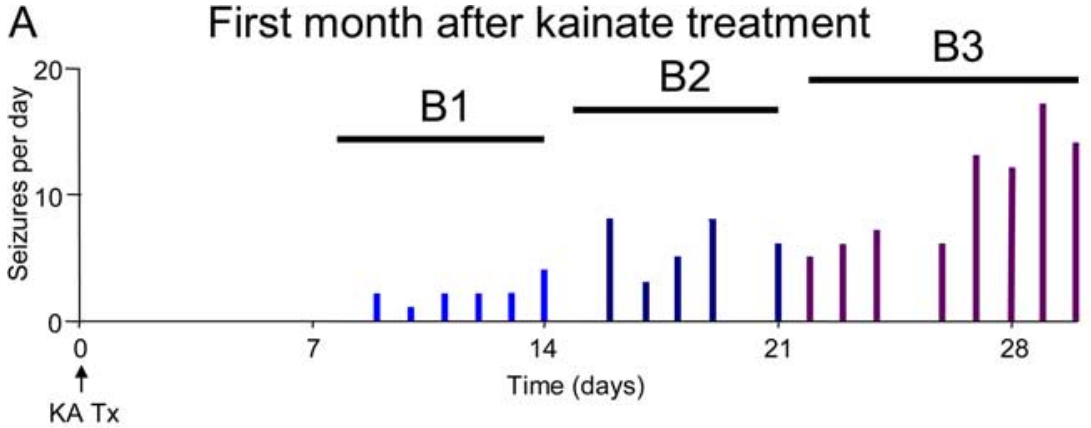

B1

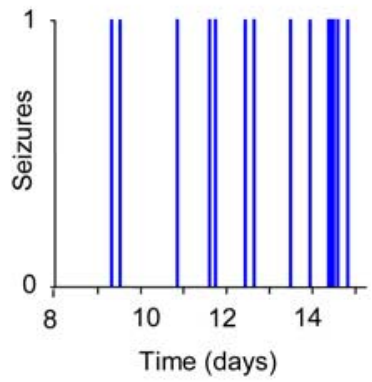

B2



B3

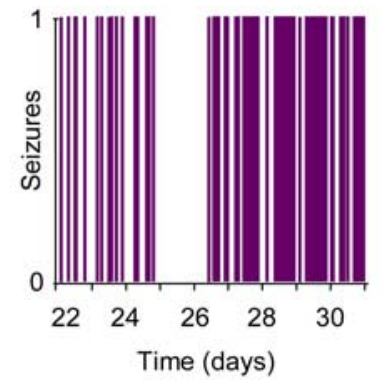

C1 Day 28 after KA

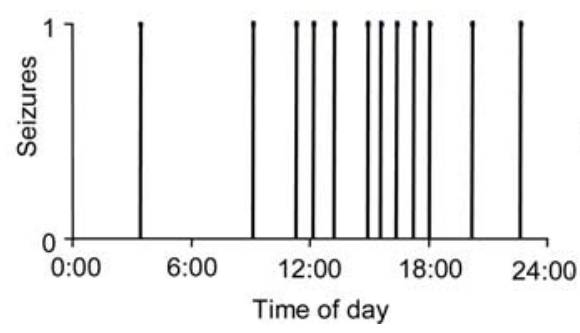

C2 Day 87 after KA

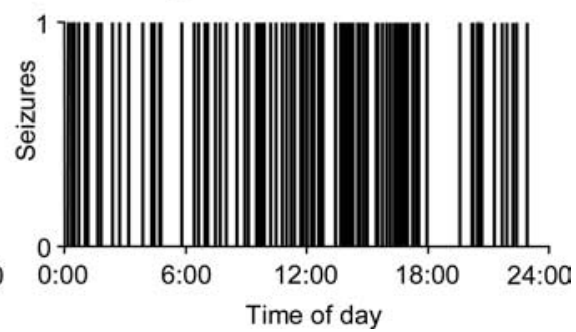

Figure 3. Raster plots of electrographic seizure frequency from an individual animal for the first month $(\boldsymbol{A})$ and for different 1 week $(\boldsymbol{B} 1-B 3)$ or $1 \mathrm{~d}(\boldsymbol{C} 1, \boldsymbol{C})$ periods. The data show the typical increase in seizure frequency and the occurrence of seizure clusters throughout the $100 \mathrm{~d}$ recording period in an individual animal. $A$, Daily seizure frequency for the first $30 \mathrm{~d}$. B1-B3, Raster plots to illustrate the occurrence of seizures and the interseizure intervals for the second (B1), third (B2), and fourth (B3) weeks [no seizures were seen during the first week (i.e., the latent period for electrographic seizures)]. C1, C2, A24 h period at day 28 (C1) and day 87 (C2) after kainate treatment. These data from a single animal illustrate the time-dependent increase in seizure frequency and the occurrence of seizure clusters (i.e., short interseizure intervals). The clusters began to occur relatively soon after the onset of spontaneous recurrent seizures, and continued throughout the $100 \mathrm{~d}$ recording period.

no significant correlation was found between the latent period to the first electrographic seizure and the longest interseizure interval $\left(r^{2}=0.47 ; p=0.06\right)$. This result indicates that the latent period to the first electrographically recorded seizure was similar across the population of kainate-treated rats to the longest interseizure interval observed during the early stages of epileptogenesis, which implies that the onset of epileptogenesis [as assessed by measuring the time after kainate-induced status epilepticus of the initial spontaneous recurrent electrographic seizures (i.e., both nonconvulsive and convulsive)] was gradual or virtually continuous. This finding does not challenge the concept that an actual latent period exists, but it does support the hypotheses that (1) the progression of epileptogenesis is a "continuous process," and (2) the duration of the latent period is a suboptimal measure of epileptogenesis. However, the shortest interseizure intervals (Fig. $4 \mathrm{~B}$ ) were as short as $1-4 \mathrm{~h}$, nearly two orders of magnitude less than the longest intervals, which indicates that the initial spontaneous recurrent seizures often occurred in clusters (i.e., interseizure intervals of at least a few days separated by intersei- zure intervals of only a few hours). Thus, in the comparison of the latent periods and the longest interseizure intervals, the longer interseizure intervals conceptually and practically represent the "intercluster intervals," whereas the shorter interseizure intervals represent the "intracluster intervals," as will be clarified in subsequent sections.

Plots of successive interseizure intervals (i.e., the reciprocals of instantaneous seizure frequency) for the first 20 seizures did not reveal clear evidence of a progressive increase in seizure frequency (Fig. $4 B)$. To better assess the temporal characteristics of the onset of epileptogenesis over a longer period of time, the number of electrographically recorded seizures per day was determined, and the daily seizure frequency was calculated over the $100 \mathrm{~d}$ period after kainate treatment (Fig. 5A; supplemental Fig. 2, available at www.jneurosci.org as supplemental material). Previous studies, based on regular but discontinuous behavioral monitoring of convulsive motor seizures, suggested that seizure frequency generally increases in this model for several months but then reaches a steady state or plateau (Hellier et al., 1998). Although a sigmoid curve best fit the average increase in electrographically recorded seizure frequency after kainate treatment (i.e., group data) (Fig. 5A), similar plots of seizure frequency versus time were more variable for individual animals (Fig. 5B,C). A Boltzmann sigmoid curve modeled the timedependent increase in seizure frequency in six of nine rats by $100 \mathrm{~d}$ after kainateinduced status epilepticus and extended to $114 \mathrm{~d}$ in one rat (supplemental Fig. 2, available at www.jneurosci.org as supplemental material). The equation describing this relationship had the following form:

$$
\text { Szrate }=\text { Inrate }+\frac{\text { Finrate }- \text { Inrate },}{\frac{1 / 2 \max -1}{\text { slope }} 1+e}
$$

where "Szrate" was the EEG seizure rate at time $t$, "Inrate" was the initial EEG seizure rate, "Finrate" was the EEG seizure rate at the plateau phase, " $1 / 2 \max$ " was the time at which the EEG seizure rate reached half-maximum, and "slope" was the maximum slope of the curve. An exponential growth curve provided the best fit to the data in the other three rats (supplemental Fig. $2 A, B, H$, available at www.jneurosci.org as supplemental material); however, a sigmoid growth curve could also be well fit to these data if one assumed that the EEG seizure frequency eventually reached a plateau, which was considered a reasonable assumption given previous data (Hellier et al., 1998, their Fig. 2) and the view that an exponential growth in EEG seizure frequency without some type of plateau phase seems untenable (in some animals, the end of the exponential growth phase was equivalent to $12-24 \mathrm{~h}$ peri- 
A



B

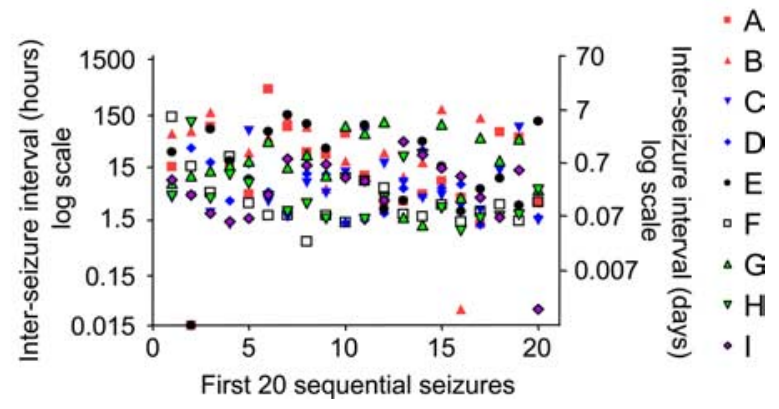

Figure 4. Similarity of the latent periods and the longest early interseizure intervals after kainate-induced status epilepticus. $A$, A plot of the latent periods for convulsive motor seizures (motor seizures with video-EEG documentation), nonconvulsive electrographically recorded seizures (i.e., EEG seizures without a convulsion), and the longest subsequent interseizure interval of the first 20 electrographically recorded intervals for each of the nine animals. The horizontal bars indicate the mean values, and the vertical bars show the SDs. These data show that the longest interseizure interval (see $\boldsymbol{B}$, below) after the first motor seizure was comparable with the latent period for electrographic seizures, which provides one line of evidence for the hypothesis that the onset of epileptogenesis is a continuous function of time. $\boldsymbol{B}$, The interseizure intervals for the first 20 electrographically recorded seizures for individual rats. This figure demonstrates the high variability in interseizure intervals in the early period of seizure progression, in which several of the long interseizure intervals were comparable with the latent period for the first electrographic seizure, and the short interseizure intervals represent seizure clusters. Each symbol and color represents an individual animal for $\boldsymbol{A}$ and $\boldsymbol{B}$ (see right side of panel).

ods of status epilepticus). The ability to fit the progression of EEG seizures over time to a sigmoid curve allowed us to measure parameters of EEG seizure progression (i.e., the slope factor and the time required to reach the half-maximum of the sigmoid curve). A correlation analysis between the number of days to the first detected EEG seizure and the first convulsive motor seizure (i.e., measures of latent period) and the two measures of EEG seizure progression were performed on those animals that could be best fit by a sigmoid curve. The EEG latent period did not significantly correlate with either the half-maximum $\left(r^{2}=0.73 ; p=0.09 ; n=\right.$ 6 ) or the slope $\left(r^{2}=0.17 ; p=0.4 ; n=6\right)$. The latent period to the first motor seizure also did not correlate with the half-maximum time $\left(r^{2}=-0.12 ; p=0.8 ; n=6\right)$, although the latent period to the first convulsive motor seizure did have a significant negative correlation with the slope $\left(r^{2}=0.72 ; p=0.03 ; n=6\right)$. Furthermore, correlations between the amount of time in status epilepticus and either the half-maximum or the slope of EEG seizure progression $\left(r^{2}=0.23, p=0.4 ; r^{2}=0.26, p=0.37\right.$, respectively), and between the total number of convulsive motor seizures during kainate treatment and either the half-maximum or the slope of EEG seizure progression were not significant $\left(r^{2}=0.47, p=\right.$ $0.3 ; r^{2}=0.55, p=0.25$, respectively). Therefore, the key findings were that (1) nonconvulsive electrographic seizures usually began after a relatively short latent period ( $\sim 1$ week), (2) convulsive motor seizures generally began to occur after the appearance of
A

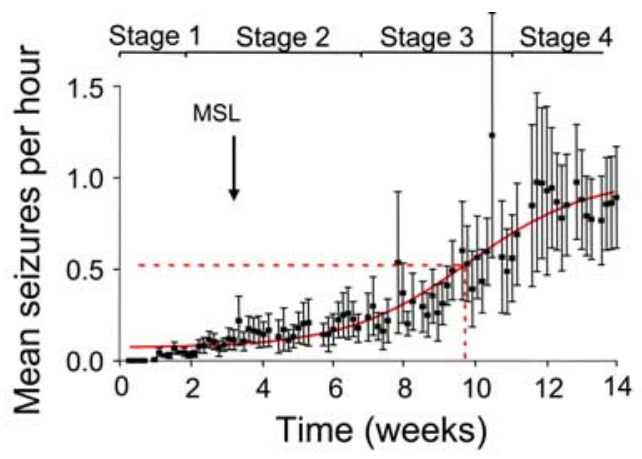

B

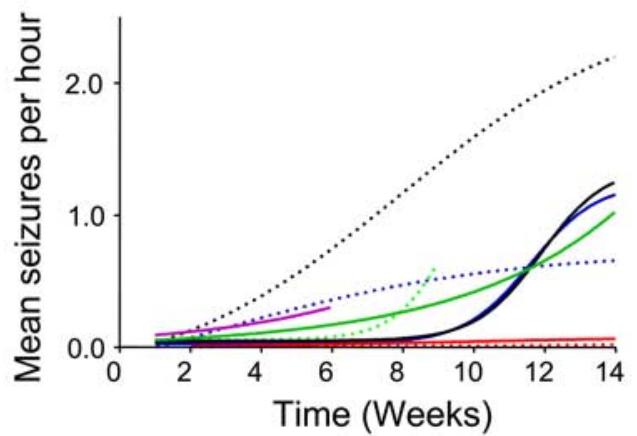

C



Figure 5. Seizure frequency as a function of time after kainate-induced status epilepticus. $\boldsymbol{A}$, The mean frequency of the electrographically recorded seizures for the group of nine kainatetreated rats increased as a function of time after status epilepticus. A sigmoid curve best described the overall time-dependent increase in seizure rate. The dashed red line shows the time to reach the half-maximum point on the sigmoid curve. Based on the sigmoid curve, the increase in seizure frequency could be divided into four stages. Stage 1 was the latent period for electrographic seizures (note: the MSL arrow denotes the mean latent period for convulsive motor seizures), and stage 2 was the period of a slow, progressive increase in seizure rate (i.e., the slow growth phase), which was followed by the exponential growth phase in seizure rate (stage 3). The plateau phase (stage 4) was the final steady-state seizure rate found in some animals. Although the plateau phase was not seen in all rats (see $\boldsymbol{B}$ and $\boldsymbol{C}$ below) (supplemental Fig. 2, available at www.jneurosci.org as supplemental material), this was likely attributable to the limited duration of the monitoring (i.e., $100 \mathrm{~d}$ ). In the repeated, low-dose kainate model, previous data suggest that most rats generally do not reach a steady state in seizure frequency for several months (Hellier et al., 1998), but some animals ultimately have such a high seizure frequency that they are often in status epilepticus. $B, C$, Plot of the data of actual seizure frequency $(\boldsymbol{B})$ and normalized seizure frequency $(\boldsymbol{C})$ from the nine individual animals $(A-I)$ that comprised the group data shown in $\boldsymbol{A}$ [the mean and SE of seizure frequency of each animal is shown in supplemental Fig. 2 (available at www.jneurosci.org as supplemental material)]. The data shown in Figure 3 were recorded from rat $D$.

nonconvulsive EEG seizures, (3) spontaneous recurrent seizures continuously increased in frequency with time after the latent period, (4) nearly all animals experienced an exponential growth in EEG seizure frequency at some point after kainate treatment, and (5) the latent periods (i.e., to nonconvulsive electrographic 
and convulsive motor seizures) did not correlate well with measures of seizure progression.

Because a Boltzmann sigmoid curve could model the group mean increase in electrographically recorded seizure frequency (i.e., both nonconvulsive and convulsive seizures) for the population of kainate-treated rats (Fig. 5A) ( $F$ test for goodness of fit, $p=0.017 ; n=9$ ), four stages of EEG seizure progression were apparent. The first distinguishable stage of EEG seizure progression was the latent period, during which no seizures were detected. Although the occurrence of the first clinical seizure is usually considered to be the end of the latent period, the observation of the first electrographically recorded seizure was viewed as the end of the first stage, or seizure-free time. The second stage began with a slow growth phase, which was characterized by a low but gradually increasing EEG seizure rate with highly variable interseizure intervals (Figs. $3 B, 4 B, 5 B, C$ ). In this second phase, the longest interseizure intervals were $4-8 \mathrm{~d}$ (see above) and nearly as long as the latent periods to the first EEG seizure. Although this phase had a positive slope, the increase in seizure frequency in some animals was barely detectable for as much as 2 months (Fig. $5 B, C$; supplemental Fig. 2, available at www.jneurosci.org as supplemental material). This result suggests that some of the rats with kainate-induced epilepsy would have been described as "nonprogressive" (Gorter et al., 2001), if they had only been subjected to 2 months of continuous monitoring, and if quantitative measures of seizure frequency had not been used. The end of the slow growth phase for each rat was arbitrarily defined as the first day in which the daily seizure rate was $\geq 0.2$ electrographically recorded seizures per hour or five EEG seizures per day. The mean number of days (for all kainatetreated rats) after treatment to obtain this EEG seizure rate was $49 \pm 8.5$ (Fig. $5 A$ ). Stage 3 of EEG seizure progression consisted of an exponential growth phase in which the number of daily EEG seizures rapidly increased (Fig. $5 A$ ). The end of this stage was mathematically defined as the time in which the fitted sigmoid curve attained $95 \%$ of its maximal value. The mean time to the end of the exponential growth phase was $79 \pm 10 \mathrm{~d}$ (Fig. 5A). Stage 4 was termed the plateau phase, in which the EEG seizure rate remained relatively stable (Fig. $5 A$ ) with a mean EEG seizure rate over this time period of $0.87 \pm 0.02 \mathrm{EEG}$ seizures per hour (i.e., $\sim 21$ seizures per day) and a short mean interseizure interval. These data show that the progression of the daily EEG seizure rate for individual rats was variable, but ultimately all animals experienced a continuous exponential rise in EEG seizure frequency. These data also indicate that the EEG latent period may best be viewed as the first of several long interseizure intervals, which eventually become shorter as the EEG seizure frequency continuously increases with time.

\section{Seizure clusters}

Clustering of seizures in this model and the pilocarpine model of status epilepticus has previously been observed (Grabenstatter et al., 2005; Goffin et al., 2007) but not quantified over long periods. Seizure clusters were either readily observable as distinct entities or could be seen as slow increases and decreases in seizure frequency (i.e., they could appear as a "waxing and waning" of the seizures). During the first month after kainate treatment, EEG seizures appeared to occur in multiples on the same day (i.e., short interseizure intervals) and often within a close time frame of each other (Figs. 3 B, C1, 4B). A Poisson distribution describes the probability of events (e.g., EEG seizures) occurring over a fixed period of time (e.g., $100 \mathrm{~d}$ ) if these events occur at a known average rate (i.e., mean EEG seizure frequency) and are indepen- dent of the time since the last event (i.e., random or nonclustering). Thus, EEG seizures that occur randomly should have a distribution of interseizure intervals that are fit by a Poisson distribution (Binnie et al., 1984), and the actual distribution of interseizure intervals should not be significantly different from randomly generated intervals with identical mean values. Clustering of the EEG seizures (i.e., nonrandom occurrence with interseizure intervals dependent on each other) would make the distribution of the interseizure intervals differ significantly from a Poisson distribution and randomly generated intervals with identical means. Statistical analyses of interevent intervals for detection of the presence of clusters have been referred to as tests of the uniformity hypothesis and the random-position hypothesis (Bock, 1985; Jain and Dubes, 1988, respectively). These analyses rely on statistical differences from a Poisson distribution and from matched, randomly generated data. To test the hypothesis that EEG seizures were occurring randomly, a Poisson function was generated for each stage, and random intervals were generated around the mean interseizure interval for each individual animal during each stage of EEG seizure progression (see Materials and Methods). The Kolmogorov-Smirnov test was used to test for differences in the cumulative distribution function between the actual interseizure intervals, the Poisson distribution, and the randomly generated intervals. In each of the different stages of EEG seizure progression, the interseizure intervals were significantly different from the Poisson distribution [stage 2, $p<$ $0.0001, n=9$ (Fig. 6 A); stage $3, p<0.0001, n=9$; stage $4, p<$ $0.0001, n=6$ ] and from randomly generated intervals (stage 2, $p<0.002, n=9$; stage 3, $p<0.0001, n=9$; stage $4, p<0.0001$, $n=6$ ). Figure $6 \mathrm{~A}$ illustrates the distribution of interseizure intervals during the slow growth phase on two scales of the ordinate ( $A 1,0-160$ intervals; $A 2,0-15$ intervals); Figure $6 A 1$ shows that $>160$ interseizure intervals (i.e., the first bin) during the slow growth phase were $\leq 250 \mathrm{~min}$ (or $\sim 4$ h; i.e., the bin duration), whereas Figure $6 A 2$ shows a peak to the right in which $>25$ intervals were between $\sim 2000$ and $6000 \mathrm{~min}$ ( or $\sim 1-4 \mathrm{~d}$ ). Therefore, this analysis of the distribution of interseizure intervals during the slow growth phase suggests that many of the interseizure intervals during a cluster were $<4 \mathrm{~h}$ and many of the intercluster intervals were $\sim 1-4 \mathrm{~d}$. Qualitatively similar relationships for the interseizure intervals were present during stages 3 and 4 (Fig. $3 C 2$ ), but the family of intervals showed a distinct shortening that corresponded to the overall higher seizure frequency. The distributions for the plateau phase used only six of the nine rats, because three animals did not achieve a plateau phase before termination of the recording. These data reject the hypothesis that the electrographically recorded seizures were simply occurring in a random manner and, instead, support the hypothesis that the seizures occur in clusters and that an increase in EEG seizure clustering may contribute to the time-dependent increase in EEG seizure frequency. These data also indicate that EEG seizure clusters were a likely source of variability throughout all phases of seizure progression, contributing to the variable rates of seizure progression seen in individual animals in Figure 5, $B$ and $C$ (i.e., the intraanimal variability).

As the EEG seizure rate progressively increased, EEG seizures tended to occur in multiple clusters with very short interseizure intervals during any given $24 \mathrm{~h}$ period (Fig. 3B1-B3; compare C2, C1).

One definition of a seizure cluster in humans is three or more motor seizures per day (Haut et al., 1999), and this measure applied to EEG seizures showed a clear increase over time after kainate-induced status epilepticus (data not shown); however, 
this measure did not seem appropriate in this model because of the relatively high overall EEG seizure rate, and no clear progression pattern in EEG seizure clusters was seen when this measure was normalized to the EEG seizure frequency (Fig. $6 B 1)$. Because many of the shortest interseizure intervals were measured to be $\sim 1 \mathrm{~h}$ soon after the onset of spontaneous recurrent seizures (Fig. $4 B$ ), and because these $1 \mathrm{~h}$ interseizure intervals represented obvious seizure clusters when separated by intercluster intervals that could be as long as a few days, we determined the frequency of occurrence of interseizure intervals that were $\leq 1 \mathrm{~h}$ as a function of time after kainate-induced status epilepticus. When the number of $\leq 1 \mathrm{~h}$ interseizure intervals per day (i.e., defined as a seizure cluster) was normalized to the daily EEG seizure frequency to control for the effect of increasing EEG seizure frequency on the analysis (Fig. 6B2) $(n=9)$, the increase in seizure clusters over time closely followed the pattern of EEG seizure progression (Fig. 5A). A Boltzmann sigmoid curve ( $F$ test, $p=0.02$ ) could be fit to this progressive increase in seizure clusters (i.e., interseizure intervals of $\leq 1 \mathrm{~h}$ ), and this function began a steep increase between weeks 5 and 7 after kainate treatment, which coincided with the beginning of the exponential growth phase (i.e., stage 3 ). Thus, this analysis of $\leq 1 \mathrm{~h}$ interseizure intervals, as a measure of seizure clusters, showed that the exponential increase in EEG seizure frequency occurred concurrently with an enhanced likelihood of electrographically recorded seizure clusters.

\section{Discussion}

The latent period, progressive increases in seizure frequency, and

\section{seizure clusters}

The key results of this study are as follows: nonconvulsive electrographic seizures almost always preceded the first convulsive motor (i.e., clinical) seizure (Bertram and Cornett, 1993, 1994); the longest interseizure interval after the first clinical seizure was nearly as long as the latent period to the first electrographically recorded seizure (Fig. $4 \mathrm{~A}$ ); in the initial period after the onset of spontaneous recurrent seizures, the interseizure intervals were highly variable (i.e., spanned 2 log units) (Fig. $4 \mathrm{~B}$ ); individual and group data indicated that a Boltzmann sigmoid function best fit the data on seizure frequency versus time after status epilepticus (Fig. 5); plots of the number of intervals as a function of the duration of the interseizure interval did not fit a Poisson distribution (i.e., were nonrandom) and had two peaks during the early, slow growth phase of epileptogenesis (Fig. $6 A$ ); and a graph of the number of interseizure intervals that were $\leq 1 \mathrm{~h}$ (normalized for seizure frequency) increased as a function of time after kainate-induced status epilepticus (Fig. 6B). These data support the hypothesis that the early phase of epileptogen-
A2

Slow growth phase



B2

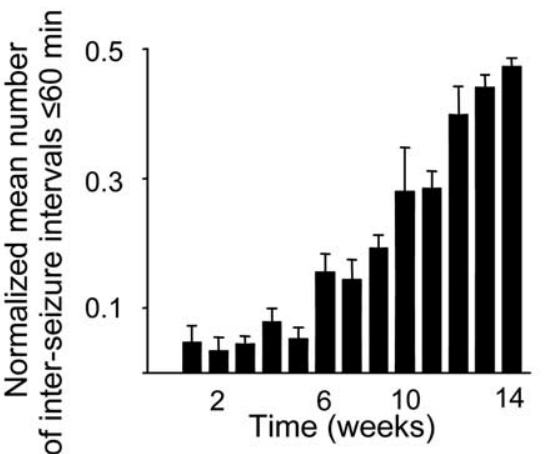

Figure 6. Seizure clusters. $\boldsymbol{A}$, Frequency histograms of the interseizure intervals for the group of nine kainate-treated rats during the slow growth phase ( $\boldsymbol{A} 2$ is a vertical expansion of $\boldsymbol{A} 1)$ show two peaks. The first bin ( $<250 \mathrm{~min}$; or $\sim 4 \mathrm{~h}$ ) had the largest number of the interseizure intervals, and the number of interseizure intervals per bin was progressively smaller for longer time periods during the slow growth phase (A1). Many of these short intervals were "within-cluster" seizures. A second peak in the frequency. Many of these longer interseizure intervals represent "intercluster" intervals. $\boldsymbol{B}$, Mean number of potential clusters (ized for seizure frequency) as a function of time after kainate-induced status epilepticus. In $\boldsymbol{B} 1$, the number of days per analyzed in B2.B1, Although the mean number of days per week in which three or more seizures was observed [i.e, a definition of seizure clusters used in human clinical studies (Haut et al., 1999)] increased as a function of time after kainate-induced status epilepticus, no progressive increase in seizures was seen when the data were normalized to the weekly seizure frequency, which indicates that this definition of seizure clustering was problematic in the kainate model because of the high seizure frequency. $\mathbf{B 2}$ (defined here as one measure of a seizure cluster), normalized to the daily seizure rate [i.e., the number of interseizure intervals of $\leq 1 \mathrm{~h}$ (i.e., seizure clusters) per day was divided by the number of seizures per day], slowly increased until week 10 .

esis is a smooth, continuous process whereby seizure frequency (and thus, seizure probability) slowly increases with time, but seizure clusters can obscure the progression. The data do not support the hypothesis that seizure probability is a step function of time after a brain insult (Fig. 1).

\section{The latent period: Is it determined by increasing seizure probability?}

These data offer a new perspective of the latent period. Backward extrapolation from the slow growth phase of epileptogenesis (i.e., the second stage) to the latent period (i.e., the first stage) suggests that the latent period is a time of slowly but continuously increasing seizure probability (Fig. 5A). Because our data suggest that seizure frequency/probability gradually increases after a brain insult, the first nonconvulsive EEG seizure and the first convulsive motor seizure are likely probabilistic events. That the EEG seizure 
latent periods appear only slightly longer than the longest interseizure intervals during the early growth phase (Fig. $4 A$ ) further supports this hypothesis. Thus, the latent period for the first EEG-recorded seizure may be viewed as the longest of the electrographically recorded interseizure intervals that occur early in the process of epileptogenesis, in which all of the longer interseizure intervals (i.e., not the short intervals that comprise seizure clusters) (see below) represent a time-dependent continuously increasing probability of the occurrence of seizures after status epilepticus. This result leads to the hypothesis that the rate of increasing seizure probability determines the duration of the latent period; and thus, the latent period may be viewed hypothetically as the time point when the increasing seizure probability asymptotically departs from a steady baseline of low seizure probability. In conclusion, therefore, the latent period may poorly reflect the epileptogenic increase in seizure probability as a function of time and may not be an appropriate measure of epileptogenesis because (1) theoretically, an asymptotic interception is difficult to measure accurately, and (2) practically, measurement requires continuous electrographic recording until the first seizure.

\section{Seizure frequency versus time: step function or sigmoid function?}

At least two key results from previous work suggest that a simple step function is inadequate to describe acquired epileptogenesis: (1) nonconvulsive electrographic seizures (often several) generally precede convulsive motor seizures (Bertram and Cornett, 1993, 1994), and (2) progressive increases in the frequency of spontaneous recurrent seizures can often be detected after status epilepticus (Bertram and Cornett, 1993, 1994; Hellier et al., 1998; Gorter et al., 2001). Because of the quantitative analyses of the longest interseizure intervals after the latent period (Fig. 4) and the plots of seizure frequency versus time (Fig. 5) from continuous recording of the spontaneous seizures, our data emphasize that the transition from the latent period to the slow growth phase is best modeled as a smooth function. This hypothetical reasoning further argues that the presence of seizure clusters (see below) obscures this smooth transition from the latent period to the slow growth phase, and seizure clusters can also obscure the exponential growth phase (see below). Bertram and Cornett (1994) also noted that the increase in seizure frequency could be quite variable, and our data suggest that an important source of variability is seizure clusters (see below). Gorter et al. (2001) emphasized the presence of progressive and nonprogressive animals; although all of the kainate-treated rats in the present study ultimately showed a progressive increase in seizure frequency, some animals spent prolonged periods ( $\sim 2$ months) with a relatively steady seizure frequency (i.e., appeared "nonprogressive") (Fig. 5B,C; supplemental Fig. 2, available at www.jneurosci.org as supplemental material), and then later underwent a profound increase in seizure frequency over a few weeks. Bertram and Cornett (1994) found that seizure frequency ultimately reached a plateau; a distinct plateau phase was not always seen in the present data, presumably because the recordings were conducted for only $100 \mathrm{~d}$, and previous data using discontinuous behavioral monitoring suggest that more time after status epilepticus is required for the plateau (Hellier et al., 1998). Therefore, a Boltzmann sigmoid curve with a slow early increase in seizure frequency followed by an exponential growth phase and a plateau phase overall provided the "best fit" as a model of the observed changes in seizure frequency over time. Two parameters characterize the sigmoid function of seizure progression: (1) the time to reach $50 \%$ of the maximum seizure frequency and (2) the slope factor, which measures the maximum increase in seizure rate. These parameters provide relatively straightforward measurements of the time course of seizure progression, from the latent period to the time of maximal maintained seizure frequency. Furthermore, these measures can be obtained using short infrequent observations; if the plateau seizure frequency is also measured, the Boltzmann function can be fit and used to provide a description of the initial time course, which removes the need for continuous EEG analysis of the period of very low seizure probability.

Although the data presented in this study do not support the hypothesis that the latent periods for either the nonconvulsive EEG seizures or the convulsive motor seizures are directly related to the progressive increase in seizure frequency, this may reflect the inherent conceptual and technical problems with the latent period described above.

\section{Seizure clusters}

The evidence for seizure clusters comes from several parts of the data set. The early interseizure intervals were both extremely long and quite short (Fig. 4B), and appeared to represent the early onset of seizure clustering, which was better documented by the observation of a non-Poisson (i.e., nonrandom) distribution of interseizure intervals (Fig. 6A). Individual variability can obviously confound analyses of seizure progression and can prevent statistical analyses that assume randomness (e.g., a normal distribution). Bertram and Cornett (1994) noted that seizure rates can be quite variable not only across animals but also through time in the same animal; it is unclear how much of the variability is attributable to seizure clusters. This variability can confound analyses of the effects of hypothetical therapeutic interventions. Whether seizure clustering contributed mechanistically to the exponential increase in seizure frequency, or was an unrelated consequence of the mechanisms of epileptogenesis, is also unclear.

\section{Is the latent period and progressive increase in seizure frequency model dependent?}

Whether the progressive increase in seizure rate is common to all types and models of acquired epileptogenesis (including humans), or may simply reflect a particular animal model, also requires additional investigation. Some studies in models of TLE have not observed progressive increases in seizure rate (Gorter et al., 2001; Mazarati et al., 2002; Bragin et al., 2004), but our data strongly suggest that both continuous and prolonged seizure monitoring is required to detect the progressive increase in seizure frequency. A recent study using discontinuous monitoring on pilocarpine-treated rats (Jung et al., 2007) reported the presence of seizures during the first week after status epilepticus, suggesting the lack of a latent period in this model. Our own recent observations suggest that the electrographic status epilepticus after pilocarpine treatment is far more intense than with the repeated, low-dose kainate model (Dudek et al., 2002, 2005), and the pilocarpine model may be more prone to having subsequent seizures in the few days after intense status epilepticus (Hellier et al., 1999, their Fig. 3, particularly B). Thus, additional work is required to determine the generality of the concepts described here.

\section{Conclusions}

These data suggest several possible conclusions concerning acquired epileptogenesis: (1) seizure probability increases continu- 
ously after a brain insult until a steady state is achieved; (2) the latent period does not define the duration of epileptogenesis and is probably a poor measure of it; (3) seizure clusters can obscure a progressive increase in seizure frequency, and yet they may contribute to increases in seizure frequency; and (4) the fitting of seizure rates after a brain insult with a sigmoid curve may be useful for future antiepileptogenesis studies. Why some animals have a nonprogressive period, why clusters occur, and why seizure frequency reaches a plateau remains unclear. Understanding the mechanisms that contribute to the slow continuous buildup in spontaneous recurrent seizures and the clustering of seizures may provide important therapeutic insights into potentially altering the outcome of patients with acquired epilepsy.

\section{References}

Annegers JF, Hauser WA, Coan SP, Rocca WA (1998) A population-based study of seizures after traumatic brain injuries. N Engl J Med 338:20-24.

Ben-Ari Y (1985) Limbic seizure and brain damage produced by kainic acid: mechanisms and relevance to human temporal lobe epilepsy. Neuroscience 14:375-403.

Bertram EH (1997) Functional anatomy of spontaneous seizures in a rat model of limbic epilepsy. Epilpesia 38:95-105.

Bertram EH, Cornett JF (1993) The ontogeny of seizures in a rat model of limbic epilepsy: evidence for a kindling process in the development of chronic spontaneous seizures. Brain Res 625:295-300.

Bertram EH, Cornett JF (1994) The evolution of a rat model of chronic spontaneous limbic seizures. Brain Res 661:157-162.

Bertram EH, Zhang DX, Mangan P, Fountain N, Rempe D (1998) Functional anatomy of limbic epilepsy: a proposal for central synchronization of a diffusely hyperexcitable network. Epilepsy Res 32:194-205.

Binnie CD, Aarts JH, Houtkooper MA, Laxminarayan R, Martins da Silva A, Meinardi H, Nagelkerke N, Overweg J (1984) Temporal characteristics of seizures and epileptiform discharges. Electroencephalogr Clin Neurophysiol 58:498-505.

Bock HH (1985) On some significance tests in cluster analysis. J Classif 2:77-108.

Bower MR, Buckmaster PS (2008) Changes in granule cell firing rates precede locally recorded spontaneous seizures by minutes in an animal model of temporal lobe epilepsy. J Neurophysiol 99:2431-2442.

Bragin A, Wilson CL, Almajano J, Mody I, Engel J Jr (2004) High-frequency oscillations after status epilepticus: epileptogenesis and seizure generation. Epilepsia 45:1017-1023.

Buckmaster PS, Dudek FE (1997) Neuron loss, granule cell axon reorganization, and functional changes in the dentate gyrus of epileptic kainatetreated rats. J Comp Neurol 385:385-404.

Dudek FE, Sutula TP (2007) Epileptogenesis in the dentate gyrus: a critical perspective. Prog Brain Res 163:755-773.

Dudek FE, Hellier JL, Williams PA, Ferraro DJ, Staley KJ (2002) The course of cellular alterations associated with the development of spontaneous seizures after status epilepticus. In: Do seizures damage the brain? (Sutula T, Pitkanen A, eds), pp 53-65. Amsterdam: Elsevier.

Dudek FE, Clark S, Williams PA, Grabenstatter H (2005) Kainate-induced status epilepticus: a chronic model of acquired epilepsy. In: Models of seizures and epilepsy (Sutula T, Pitkanen A, eds), pp 415-432. New York: Elsevier.

French JA, Williamson PD, Thadani VM, Darcey TM, Mattson RH, Spencer SS, Spencer DD (1993) Characteristics of medial temporal lobe epilepsy: I. Results of history and physical examination. Ann Neurol 34:774-780.

Fuerst D, Shah J, Kupsky WJ, Johnson R, Shah A, Hayman-Abello B, Ergh T, Poore Q, Canady A, Watson C (2001) Volumetric MRI, pathological, neuropsychological progression in hippocampal sclerosis. Neurology $57: 184-188$.
Goffin K, Nissinen J, Van Laere K, Pitkänen A (2007) Cyclicity of spontaneous recurrent seizures in pilocarpine model of temporal lobe epilepsy in rat. Exp Neurol 205:501-505.

Gorter JA, van Vliet EA, Aronica E, Lopes da Silva FH (2001) Progression of spontaneous seizures after status epilepticus is associated with mossy fibre sprouting and extensive bilateral loss of hilar parvalbumen and somatostatin-immunoreactive neurons. Eur J Neurosci 13:657-669.

Grabenstatter HL, Ferraro DJ, Williams PA, Chapman PL, Dudek FE (2005) Use of chronic epilepsy models in antiepileptic drug discovery: the effect of topiramate on spontaneous motor seizures in rats with kainateinduced epilepsy. Epilepsia 46:8-14.

Haut SR, Shinnar S, Moshé SL, O’Dell C, Legatt AD (1999) The association between seizure clustering and convulsive status epilepticus in patients with intractable complex partial seizures. Epilepsia 40:1832-1834.

Hellier JL, Dudek FE (2005) Chemoconvulsant model of chronic spontaneous seizures. Curr Protoc Neurosci 9:9.19.

Hellier JL, Patrylo PR, Buckmaster PS, Dudek FE (1998) Recurrent spontaneous motor seizures after repeated low-dose systemic treatment with kainate: assessment of a rat model of temporal lobe epilepsy. Epilepsy Res 31:73-84.

Hellier JL, Patrylo PR, Dou P, Nett M, Rose GM, Dudek FE (1999) Assessment of inhibition and epileptiform activity in the septal dentate gyrus of freely-behaving rats during the first week after kainate treatment. J Neurosci 19:10053-10064.

Jain AK, Dubes RC (1988) Algorithms for clustering data. Englewood Cliffs, NJ: Prentice Hall.

Jung S, Jones TD, Lugo JN Jr, Sheerin AH, Miller JW, D’Ambrosio R, Anderson AE, Poolos NP (2007) Progressive dendritic HCN chanelopathy during epileptogenesis in the pilocarpine model of epilepsy. J Neurosci 27:13012-13021.

Margerison JH, Corsellis JA (1966) Epilepsy and the temporal lobes. A clinical, electroencephalographic and neuropathological study of the brain in epilepsy, with particular reference to the temporal lobes. Brain 89:499-530.

Mathern GW, Babb TL, Vickrey BG, Melendez M, Pretorius JK (1995) The clinical-pathogenic mechanisms of hippocampal neuron loss and surgical outcomes in temporal lobe epilepsy. Brain 118:105-118.

Mazarati A, Bragin A, Baldwin R, Shin D, Wilson C, Sankar R, Naylor D, Engel J Jr, Wasterlain C (2002) Epileptogenesis after self-sustaining status epilepticus. Epilepsia 43[Suppl 5]:74-80.

Racine RJ (1972) Modification of seizure activity by electrical stimulation. II. Motor seizure. Electroencephalogr Clin Neurophysiol 32:281-294.

Sutula TP, Dudek FE (2007) Unmasking recurrent excitation generated by mossy fiber sprouting in the epileptic dentate gyrus: an emergent property of a complex system. Prog Brain Res 163:541-563.

Tasch E, Cendes F, Li LM, Dubeau F, Andermann F, Arnold DL (1999) Neuroimaging evidence of progressive neuronal loss and dysfunction in temporal lobe epilepsy. Ann Neurol 45:568-576.

Walker MC, White HS, Sander JW (2002) Disease modification in partial epilepsy. Brain 125:1937-1950.

White AM, Williams PA, Ferraro DJ, Clark S, Kadam SD, Dudek FE, Staley KJ (2006) Efficient unsupervised algorithms for the detection of seizures in continuous EEG recordings from rats after brain injury. J Neurosci Methods 152:255-266.

Williams P, White A, Ferraro D, Clark S, Staley K, Dudek FE (2006) The use of radiotelemetry to evaluate electrographic seizures in rats with kainateinduced epilepsy. J Neurosci Methods 155:39-48.

Williamson A, Spencer SS, Spencer DD (1995) Depth electrode studies and intracellular dentate granule cell recordings in temporal lobe epilepsy. Ann Neurol 38:778-787.

Wuarin JP, Dudek FE (2001) Evidence from whole-cell recordings and focal activation of caged glutamate that excitatory synaptic input to granule cells increases with time after kainate treatment. J Neurophysiol 85:10671077. 